\title{
Current Topics in Pharmacological Research on Bone Metabolism: Regulation of Bone Mass by the Function of Endogenous Modulators of Bone Morphogenetic Protein in Adult Stage
}

\author{
Masaki Noda ${ }^{1, *}$ \\ ${ }^{I}$ Department of Molecular Pharmacology, Medical Research Institute, Tokyo Medical and Dental University, \\ 3-10 Kanda-Surugadai 2-Chome, Chiyoda-ku, Tokyo 101-0062, Japan
}

Received November 24, 2005; Accepted January 29, 2006

\begin{abstract}
Bone formation in adults determines the basic mass of bone and hence it is crucial to understand the mechanisms of its regulation. As remodeling is proceeding, bone resorption is determined by the physical conditions. Especially in women, postmenopausal bone loss is characterized by rapid bone loss due to increase in bone resorption and relative negative balance between such bone resorption and accelerated bone formation. Bone formation is also critical in considering measures to treat patients with very low bone mass. In these subjects, simple suppression of bone resorption would not be enough to maintain bone mass. Thus, bone formation in the adult is of importance in terms of both pathological and therapeutic aspects. This review addresses the possible roles of bone formation modulators in the maintenance of bone mass in the light of understanding the determination of adult bone mass.
\end{abstract}

Keywords: bone mass, bone morphogenetic protein, bone resorption, bone formation, Tob

Bone mass levels are major determinants of bone strength. After menopause in women, bone mass levels decline rapidly and reach certain levels to be susceptible to fructures even after minor impact force or without any injury. Bone mass levels are determined by the activities of two major types of cells. One type of these cells is the osteoblast and the other is the osteoclast. Osteoblasts are the cells that form bone and this activity is first observed when the body structure is being formed during embryonic life. Later on, osteoblastic activities would not cease during an individual's entire life. Osteoclastic bone resorbing activities are also involved in the very beginning of the embryonic life where bone is being formed after removal of rudiments to make particular structures of the skeleton and to adjust its shape along with growth of the body. Similar to the activities in osteoblasts, osteoclastic activities are also observed throughout life. The strengths of these activities are balanced to be equal.

In the average-sized human, approximately $500 \mathrm{mg}$ of

*Corresponding author. noda.mph@mri.tmd.ac.jp

Published online in J-STAGE: March 14, 2006

DOI: $10.1254 /$ jphs.FMJ05004X6 calcium is removed from calcified bone into the blood stream every day, and at the same time, about $500 \mathrm{mg}$ of calcium is deposited onto bone by transferring it from the blood stream due to the activities of osteoblasts to form bone. It is thought that such a fine balancing of calcium deposition and removal between bone and blood compartment is supported by relatively high buffering capacity of the body. The buffering action maintains calcium levels in the blood in a relatively narrow range $(10.5 \pm 1 \mathrm{mg} / \mathrm{dl})$.

The systemic regulation of such calcium balance is based on endocrine hormone actions of vitamin D3, calcitonin, and parathyroid hormone (PTH). Downstream to these systemic regulations are local modulators such as bone morphogenetic protein (BMP), fibroblast growth factor (FGF), insulin-like growth factor (IGF), and other peptide or non peptide compounds. Among those, BMP is the most important cytokine as it can form bone ectopic sites in the body such as muscle and subcutaneous tissues when it is implanted with a certain scafford. Since BMP is expressed not only in embryonic life but also this protein is stored in a certain quantity in adult bone, BMP has been postulated to function in adult bone. 
As BMPs are multi-functional as it works not only in bone but also in body patterning as well as in signaling for organogenesis in a number of tissues, it appears necessary to regulate this molecule properly by a variety of antagonists in various tissues. In fact, a number of BMP antagonists have been identified in a number of tissues in different spatial and temporal manners.

One of the BMP inhibitors present in osteoblasts of adult bone is Tob. Tob was first identified by us, Yoshida et al. (1), as a protein that binds to ErbB2. Since this protein suppresses proliferation of cells and it shares a homology region with several other members of the Tob family, a group of these molecules belonging to this family are regarded as anti-proliferative regulators. In addition to its function in cell proliferation, Yoshida et al. discovered that this protein binds to Smad and inhibits BMP activities.

Tob family members appear to bind to Smad or other signaling molecules and suppress their function. According to Yoshida et al., the cells obtained from the calvarial of Tob knockout mice responded to BMP treatment by increasing alkaline phosphatase expression, more than the responses observed in wild type mice calvarial cells.

In order to see whether the effects of Tob deficiency on BMP function could be seen in vivo, mice were subjected to injections with BMP onto their calvarial bone. Wild type and Tob knockout mice were used for these experiments. As previously known, BMP injections of $2 \mu \mathrm{g} /$ injection for 5 times during 10 days resulted in formation of new bone on top of the calvarial bone. As a control, saline was injected similarly onto the top of the calvarial bone and no response was observed in wild type as well as in Tob knockout mice. In contrast to BMP-treated wild type mice, Tob knockout mice produced larger new bone mass on top of the calvarial bone after 5 times injection with BMP2. Quantification indicated that the two-dimensional area of newly formed bone as well as vertical dimension were all enhanced in Tob knockout mice, indicating that the cells sitting on top of the calvarial tissue in Tob knockout mice were more responsive to BMP in terms of new bone formation (1).

We wished to examine the morphology of the cells in this newly formed bone mass. Histological sections were prepared from newly formed bones on top of the calvarial bone after BMP treatment. Interestingly, most of these newly formed bones were not continuously connected to the original calvarial bone as they were separated by several layers of soft tissue cells covering the top of the original calvarial bone surface. Separation as well as morphologies of individual bone forming osteoblasts was similar between newly formed bone produced on top of the calvaria of wild type mice injected with BMP and those newly formed bones on top of the calvaria of Tob knockout mice injected with BMP.

Since periosteal bone response to BMP is basically reflecting bone cell behaviors within cortical regions and not likely the bone marrow residing bone cell population which are basically contributing to form cancellous bone, we asked a question of whether cancellous bone populations are influenced by the modulation based on the activity of Tob. For this purpose, bone marrow ablations were conducted (2). For control wild type mice, femoral bone marrow tissues were removed by inserting a Kirchner wire with a diameter of $0.6 \mathrm{~mm}$. After insertion, the mesenchymal cells resumed, as has been already described (3). Subsequently, within 2 to 3 days after ablation, progenitor cells appear in this ablated region possibly by chemotaxis activity and then they proliferate at this site to expand their number. Between 7 to 10 days after ablation, these mesenchymal cells in the region of ablation started to differentiate into osteoblasts and matrix deposition as well as calcification could be observed histologically as well as roentgenographically. Then, from day 10 to 14 after ablation, osteoclastic cells appear very quickly within the ablated region where newly formed bone marrow is filling the ablated cavity. These osteoclasts resorb newly formed bone specifically without influencing much of the preexisting cancellous bone located side by side with the newly formed bone. The osteoclastic activities continue from about day 14 to day 20 and quickly resorb newly formed trabecular bones within the ablated marrow. This results in restoration of normal bone marrow by the end of two to three weeks. The time courses of bone regeneration and bone marrow restoration are quite reproducible. We compared formation of newly formed bone within the ablated area between the two genotypes. We found that time regeneration courses as well as patterning and amount of such newly formed bone after bone marrow ablation were similar between the two genotypes. It was previously reported that activity of cancellous bone forming cells within bone marrow, which are responsible for newly formed bone after bone marrow ablations, are under the control of various cytokines including not only BMP but also transforming growth factor $\beta$ (TGF $\beta$ ) s and FGFs. Therefore, this data indicates that Tob was specifically inhibiting the activity of BMP and not overall growth factors such as FGFs and TGF $\beta$ s. The recovery of ablated bone marrow tissues by the formation of newly formed woven bone would be under the control of cells that are regulated by comprehensive signaling based on various cytokines including TGF $\beta$ and FGF. 
Although it was observed that Tob inhibits the action of BMP by interfering with the function of the BMP signaling molecule Smad as well as transfer of the signal from the membrane to the nucleus, further elucidation of the physiological function of Tob is still necessary. Thus, we analyzed the effects of Tob on bone remodeling activities in a osteopenic model. To do this, we chose a mouse system where ovariectomy was conducted to simulate bone turnover in post menopausal women. In wild type mice subjected to ovariectomy, reduced trabecular bone mass was observed previously (4). In Tob knockout mice, basal bone mass is significantly higher than bone mass levels in wild type and sham control mice. After ovariectomy decrease in bone mass levels in Tob knockout mice was observed. However, even after reduction in bone mass due to ovariectomy, the levels of bone mass in Tob knockout mice were comparable to those in sham-operated wild type mice. Thus, significant preservation of bone mass was observed due to deficiency of Tob even after a significant bone loss due to ovariectomy (4).

In order to see whether such bone mass preservation was due to the effects of Tob deficiency on "bone formation", "bone resorption", or both, we conducted histomorphometry. Ovariectomy enhanced the levels of bone formation as known before due to the high turnover state under the estrogen-depleted condition in wild type mice. In case of Tob deficiency, basal levels of "bone formation rate" (BFR) and "mineral apposition rate" (MAR) were both higher than the basal levels of these parameters in wild type mice. After ovariectomy, there was a high turnover state occurring in Tob knockout mice. Interestingly, bone formation rate and mineral apposition rate were both higher than the levels in already enhanced bone formation activities in ovariectomized wild type mice. Thus, Tob deficiency super enhances the activity of bone formation after ovariectomy.

Next, we determined if bone resorption part is altered by the deficiency of Tob. In wild type mice, ovariectomy increased the levels of osteoclast number as well as osteoclast surface showing increase in bone resorption activities. In Tob knockout mice, the levels of bone resorption such as osteoblast number and osteoclast surface were similar to those in sham-operated wild type mice. After ovariectomy, the levels of these osteoclastic parameters based on morphometry were both increased in Tob knockout mice to levels similar to those observed in wild type mice. These observations point to the fact that Tob deficiency specifically affects bone formation but not bone resorption even in the high turnover state of estrogen-depleted mice.

We then examined the mechanism for the Tob deficiency effects on the molecular level. RNA expression levels of genes in bones of wild type mice as well as Tob knockout mice were analyzed based on RT-PCR. Type I collagen, alkaline phosphatase, and osteocalcin mRNA levels were enhanced by ovariectomy in wild type mice, reflecting the high turnover states where osteoblastic activities were enhanced. In Tob knockout mice, even sham-operated animals showed increased levels of type I collagen, alkaline phosphatase, and osteocalcin, similar to the enhancement after ovariectomy in wild type mice. Ovariectomy in Tob knockout mice resulted in super-enhancement of the mRNA expression of type I collagen and the other osteoblastic marker-related mRNAs, reflecting similar enhancement in osteoblastic parameters levels in histomorphometry and bone mass levels.

On the other hand, osteoclastic phenotype-related mRNAs such as those for RANKL (receptor activator of nuclear factor $\kappa \mathrm{B}(\mathrm{NF} \kappa \mathrm{B})$ ligand), RANK (receptor activator of $\mathrm{NF} \kappa \mathrm{B}$ ), or OPG (osteoprotegerin) were enhanced by ovariectomy in wild type mice. Basal levels of these mRNAs in Tob knockout mice were similar to those in wild type mice. The mRNA expression levels were increased after ovariectomy in Tob knockout mice. Thus, RNA expression analysis also indicated that Tob deficiency affected specifically the levels of osteoblastic activity to super enhance bone mass levels.

We also examined molecular aspects of Tob actions in modulation of estrogen depression-induced bone loss. First, we examined the effects of Tob on osteoblastic cells in terms of alkaline phosphates expression during osteoblastic differentiation. When MC3T3E1 osteoblastic cells were transfected with Tob vector to overexpress this molecule and were treated with estrogen in phenol-red-free medium, alkaline phosphates activities were enhanced by the treatment with BMP. When either estrogen or Tob was removed, alkaline phosphatase levels were increased. Further increase was observed when both estrogen and Tob were removed from this setting of BMP treatment. These in vitro observations were in parallel to what we observed in vivo in the animals with regard to bone mass as well as osteoblastic activities such as bone formation rate and mineral apposition rate.

To see whether promoter activity of BMP responsive reporter would be altered by the presence or absence of Tob, similar cell cultures as mentioned above, using MC3T3E1 cells, were transfected with Tob and cultured in the presence of estrogen in phenol-red-free medium. The difference between the previous experiment and this one is the transfection of a GCCG-reporter into these cells. Luciferase activity was enhanced by the treatment with BMP in these cells. When either estrogen or Tob 
was removed, luciferase activity was enhanced. Finally, both of the two modulators, that is, estrogen and Tob, were removed, luciferase activity was further enhanced more than the enhancement in the case of removal of one of the two. Therefore, these observations were consistent with those obtained from alkaline phosphatase experiments as well as in vivo observations, especially with regard to osteoblastic activities.

Thus, we conclude that Tob is acting via modulating BMP activities and removal of Tob suppression of osteoblastic activities is associated with estrogen actions, in that estrogen itself suppresses osteoblastic cells at least in this setting. These experimental data illustrate that in patients with postmenopausal osteoporosis, basal levels of bone mass as well as bone formation activities after estrogen depletion are suppressed by the presence of endogenous inhibitory molecules like Tob. Removal of such inhibitory activities may help increase bone volume based on activation of osteoblastic function.

Tob by itself has been observed to be under the control of BMP. In fact, microaray analysis experiments were conducted by using MC3T3E1 cells and identified genes expressed in osteoblasts. MC3T3E1 cells, compared to NIH3T3 cells, express significantly higher levels of $\mathrm{p} 53$. In these cells, expression of BMP receptor type I or TGF $\beta$ were observed in association with osteoblastic differentiation. Interestingly, Tob expression was also sharply induced in MC3T3E1 cells upon their differentiation.

Overall, these observations on inhibitory molecules against BMP indicated that endogenous modulation of osteoblastic activities is continuously present in adult bone to maintain their remodeling cycle. Removal of such inhibitory molecules like Tob would not be fatal. Removal of Noggin results in embryonic death. In contrast, in adults, bone mass inhibitors appear to be significant in bone mass regulation, but their removal would not be detrimental to the body $(3,5)$. This would further indicate the possible application of the modulators of these molecules for novel treatments for osteoporosis in the future.

\section{References}

1 Yoshida Y, Tanaka S, Umemori H, Minowa O, Usui M, Ikematsu N, et al. Negative regulation of BMP/Smad signaling by Tob in osteoblasts. Cell. 2000;103:1085-1097.

2 Usui M, Yoshida Y, Yamashita T, Tsuji K, Isao I, Yamamoto T, et al. Enhancing effect of Tob deficiency on bone formation is specific to bone morphogenetic protein-induced osteogenesis. J Bone Miner Res. 2002;17:1026-1033.

3 Morinobu M, Nakamoto T, Hino K, Tsuji K, Shen ZJ, Nakashima K, et al. The nucleocytoplasmic shuttling protein $\mathrm{CIZ}$ reduces adult bone mass by inhibiting bone morphogenetic protein-induced bone formation. J Exp Med. 2005;201:961-970.

4 Usui M, Yoshida Y, Tsuji K, Oikawa K, Miyazono K, Ishikawa I, et al. Tob deficiency superenhances osteoblastic activity after ovariectomy to block estrogen deficiency-induced osteoporosis. Proc Natl Acad Sci U S A. 2004;101:6653-6658.

5 Shen ZJ, Nakamoto T, Tsuji K, Nifuji A, Miyazono K, Komori $\mathrm{T}$, et al. Negative regulation of bone morphogenetic protein /Smad signaling by Cas-interacting zinc finger protein in osteoblasts. J Biol Chem. 2002;277:29840-29846. 